\title{
THE HAMBURG/ESO SURVEY FOR BRIGHT QSOs - SLITLESS SPECTROSCOPY AT HIGH RESOLUTION
}

\author{
L. WISOTZKI \\ Hamburger Sternwarte \\ Gojenbergsweg 112 \\ D-21029 Hamburg \\ Germany
}

\section{Introduction}

The digital revolution in the evaluation of photographic plates and the introduction of automated quasar search techniques have drastically increased the number of known QSOs over the past decade. However, most of these QSOs are so faint that their use is limited to statistical studies. The bright end of the quasar population is still dominated by objects selected in other wavebands, such as radio, X-rays, or even infrared. We have started in 1990 a wide-angle objective-prism survey (the Hamburg/ESO Survey, HES) using the $1 \mathrm{~m}$ ESO Schmidt telescope (Reimers 1990). The survey is intended to cover $5000 \mathrm{deg}^{2}$, of which about a third has been acquired up to now. The prime goal is to compile a large sample of bright $(B<17.5)$ QSOs suited for detailed followup studies, in particular for high-resolution spectroscopy. Other objectives are to search for gravitational lens candidates and to directly measure the local luminosity function of quasars. In each Schmidt field, a spectral and a direct plate are scanned with the PDS 1010G microdensitometer at Hamburg, followed by an automated candidate selection and subsequent follow-up spectroscopy with the ESO $1.52 \mathrm{~m}$ and $3.6 \mathrm{~m}$ telescopes. A novel feature in our survey is the use of an objective-prism with a dispersion of $450 \AA / \mathrm{mm}$ at $\mathrm{H} \gamma$, yielding a seeing-limited spectral resolution of 10-20 $\AA$ FWHM. A full documentation of the survey techniques is in preparation. First results include the discoveries of the second-brightest QSO in the south (Wisotzki et al. 1991), and of a bright double QSO at high redshift, probably a gravitational lens (Wisotzki et al. 1993). In this contribution I want to show how a wide-angle quasar survey like the HES can benefit from the high resolution of the survey spectra.

\section{Efficient Selection of Quasar Candidates}

The two most outstanding spectral properties of quasars at optical wavelengths are their unusual continua and the presence of strong broad emission lines, both perceptible already at very low spectral resolution. Slitless spectroscopy is an ideal tool to automatically select quasar candidates showing at least one of these properties, as was pioneered by Clowes (1981) and further developed in the LBQS (Morris et al. 1991, and references therein). If a strong broad line is lacking in the prism spectrum, which due to the restricted spectral window of the IIIa-J emulsion 
and the limited $\mathrm{S} / \mathrm{N}$ ratio is often the case, spectroscopic confirmation is needed. In the case of broad-band colour surveys, follow-up is obviously indispensable. The efficiency in the follow-up process (defined as the number of confirmed quasars divided by the number of observed candidates) depends on the magnitude limit: while only 7\% out of $1800 \mathrm{UV}$ excess objects in the Palomar-Green survey (average limit B $<16.2$ ) were quasars, fainter surveys are less heavily affected. In the case of the LBQS, an efficiency of $30-40 \%$ is an average value (Chaffee, personal communication).

The selection of QSO candidates in the Hamburg/ESO survey is essentially a two-stage process. A full spectral plate scan is performed only at significantly lower spectral resolution, thereby speeding up the plate acquisition by a factor of 5 and increasing the $S / N$ in the individual spectra. This high throughput in plate scanning is a necessary prerequisite for wide-angle work. After extraction of the spectra, a multitude of selection criteria is employed, covering broad- and narrow-band colours, emission and absorption line searches, continuum slope evaluations, and a general method to search for peculiar spectra (Wisotzki \& Groote 1992). Overlapping spectra are excluded by cross-check with the corresponding direct plate scan. The remaining candidates are scanned a second time, now with full resolution. Note that the spectral resolution of the ESO Schmidt objective-prism spectra is comparable to what is commonly employed for follow-up spectroscopy. This allows us to easily identify even narrow Balmer absorption lines in hot subluminous stars which form the most abundant contaminant in the candidate samples. Figure 1 illustrates the virtues of performing this sort of 'internal follow-up' directly on the prism plates. Initially, we evaluated these candidate spectra by eye inspection only; meanwhile a much more accurate treatment of cross-correlating each spectrum with a Balmer line template has been developed, allowing the tracing of stellar absorption features even in rather noisy spectra. We were thus able to increase the overall efficiency of our last observing run in March 1993 to 67\%, exceeding $90 \%$ for the brighter candidates.
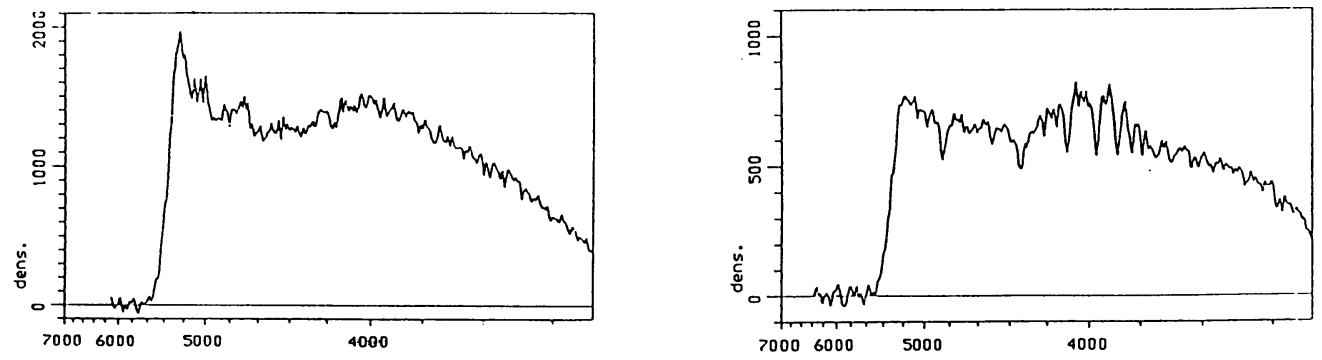

Figure 1. ESO Schmidt objective prism spectra of a low-redshift QSO (left) and a subluminous hot star of Type SdB (right). The ordinate axis shows photographic density above sky background in arbitrary units. Note the non-linear wavelength scale. The sharp edge at $5400 \AA$ is due to the emulsion sensitivity cutoff. 


\section{Towards an Optically Flux-Limited QSO Sample}

The problems of selection effects in optically selected QSO samples have been addressed by many authors. While automated techniques have brought considerable progress in that respect, it is by no means solved. A particular problem occurs at the bright end of the quasar population, where the low efficiency of colour-based searches is in hard competition with completeness. The recent discussion about incompleteness in the PG sample illustrates this (Goldschmidt et al. 1992). Ideally, one would like to take slit spectra of all objects above a given flux limit within the survey area. While that may be possible with fibre-coupled spectrographs in small deep fields, it is out of the question for wide-angle applications. However, our successful incorporation of absorption-line searches in the selection of QSO candidates using the high-dispersion ESO prism as described above has prompted us to attempt the design of a much more general definition of a 'quasar candidate'. The idea is to construct a candidate sample by eliminating all spectra that unambiguously show stellar absorption lines. A pseudo-continuum estimate is determined by subsequent application of median and Gaussian filtering together with an iterative clipping procedure for broad lines. The pseudo-continuum is subtracted, and the difference spectrum is next cross-correlated with a large number of stellar absorption line templates. The quality of a particular template fit is measured with a $\chi^{2}$ test. We have compiled a template library, presently consisting of 15 different spectral types, which is fully sufficient to match the pattern in all not too exotic stars (see Fig. 2 for illustration). Note that we do not intend to perform a real MK classification; the method only serves the purpose of separating stellar/non-stellar spectra.

If galaxies are excluded from the remaining candidates, the only significant contamination results from low-metallicity subdwarfs. Spectroscopic follow-up is certainly required. To make such an approach feasible, it is of course required to restrict the sample to spectra showing a sufficient $\mathrm{S} / \mathrm{N}$ ratio. Depending on the plate quality, the corresponding magnitude limit on the ESO objective-prism plates is around $B=16.5 \pm 0.5$. We have not yet determined the follow-up efficiency of the technique. While the classification algorithms are already fully developed and tested, we intend to incorporate this approach in the next follow-up campaign in 1994 for a large number of fields. From our tests, we estimate the efficiency to be of the order of $30 \%$ with respect to quasars. However, in addition to quasars, other unclassifiable objects will sample rare classes such as white dwarfs of types DC, DQ, or magnetic DAs, bright BL Lac objects, or even more exotic populations.

\section{Conclusions}

The Hamburg/ESO survey operates with two magnitude limits. The faint limit of $B=17.5-18$ is dictated by the efficiency with which quasar candidates can be discriminated from stars. The search criteria can be used in a very non-restrictive form, because of the 'internal follow-up' procedure that makes full use of the high spectral resolution in a second selection step and leads to candidate samples with a very low stellar contamination. Although it is unlikely that a large number of QSOs is missed this way (except possibly close to the plate limit), the criterion-based approach cannot guarantee the completeness of the sample. Note that, for the principal aim of finding QSOs suited for high-resolution spectroscopy, strict completeness is not a necessary condition.

The completeness limit of $B=16-17$ results from the ability to recognise known stellar 

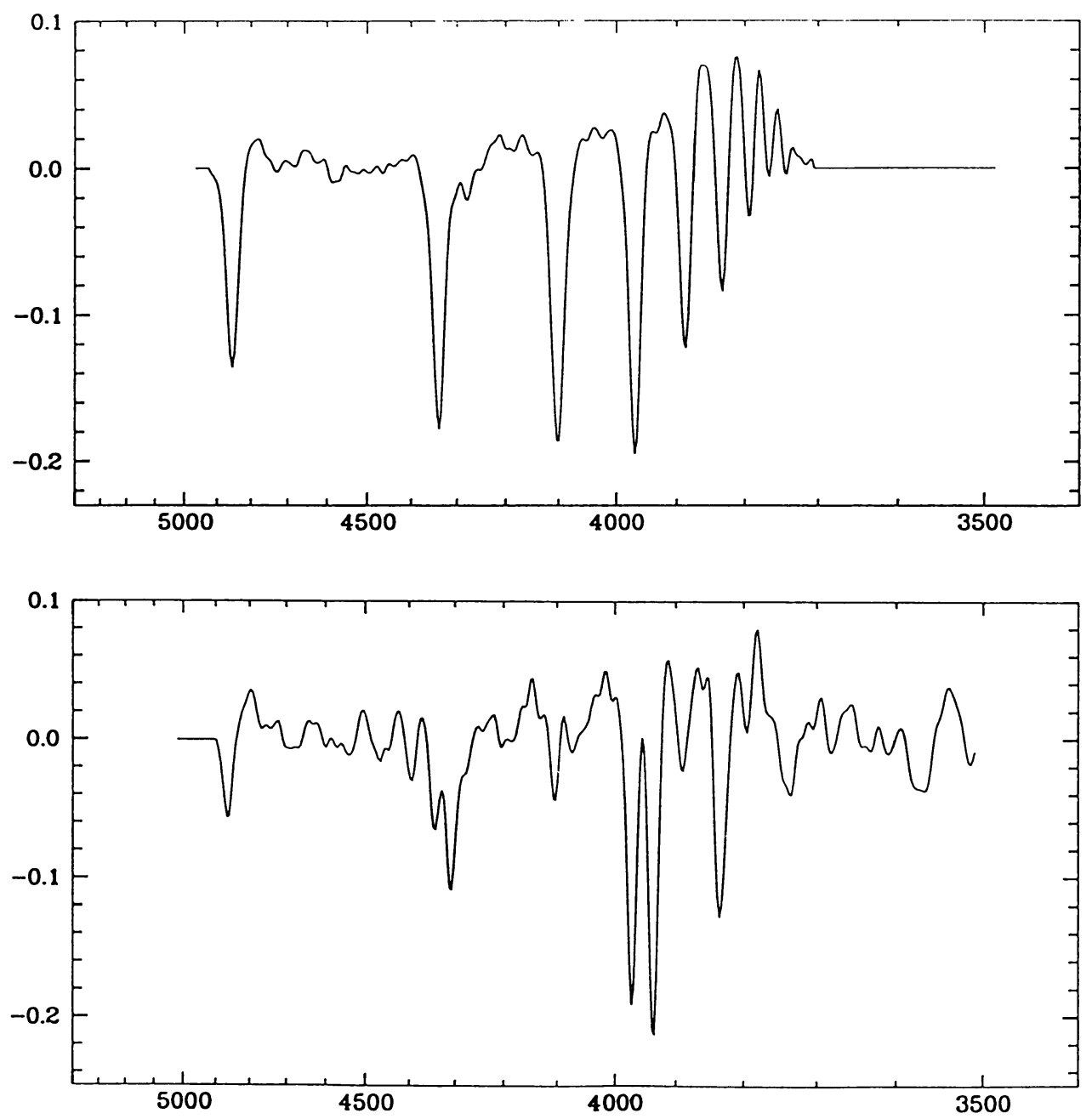

Figure 2. Sample absorption-line patterns used for the automated spectral classification. Top: A0 template, showing a pure Balmer line spectrum. Bottom: Early-type G; apart from the strong $\mathrm{Ca} \mathrm{II} \mathrm{H+K}$, several metal lines and blends are visible.

absorption features in the digital spectra with sufficient $\mathbf{S} / \mathrm{N}$. Quasar candidates in this subsample are defined by the absence of such features, independent of continuum shape. The limitations of traditional criterion-based selection techniques can thus be overcome, and we aim at constructing a bright quasar sample that is truly flux limited at optical wavelengths. Such a sample will be of eminent value for the determination of quasar evolution and other properties. 


\section{Acknowledgements}

The Hamburg/ESO survey is carried out in collaboration with D. Groote, H.-J. Hagen, T. Koehler, and D. Reimers. The plate material and follow-up observations were obtained in the course of a key programme at the European Southern Observatory at La Silla, Chile. This work has been supported by the Verbundforschung (BMFT) under grant 05-5HH41A.

\section{References}

Clowes, R.G., Cooke, J.A. and Beard, S.M., 1984. Mon. Not. R. astron. Soc., 207, 99.

Goldschmidt, P., Miller, L., LaFranca, F. and Cristiani, S., 1992. Mon. Not. R. astron. Soc., 256, 65p.

Morris, S.L., Weymann, R.J., Anderson, S.F., Hewett, P.C., Foltz, P.C., Chaffee, F.H., Francis, P.J. and MacAlpine, G.M. Astron. J., 102, 1627.

Reimers, D., 1990. The Messenger, 60, 13.

Wisotzki, L., Wamsteker, W. and Reimers, D., 1991. Astron. Astrophys., 247, L17.

Wisotzki, L. and Groote, D., 1992. In ‘4th ESO/ST-ECF Data Analysis Workshop', ESO Conf. and Workshop Proc. 41, eds. P.J. Grosbøl, R.C.E. de Ruijscher, p. 27.

Wisotzki, L., Koehler, T., Kayser, R. and Reimers, D., 1993. Astron. Astrophys., in press. 